In our case, although the donor had lived in France for 15 months and had a relatively low antibody titre, she may have had a subclinical parasitaemia with a chloroquine resistant strain (IC50 $223 \mathrm{nmol} / \mathrm{l}$ ). Although the organs were flushed out before transplantation, schizonts may have adhered to the vascular endothelium and multiplied when flushed with the recipient's blood.

The lack of knowledge about the donor's origin combined with the many other possible causes of fever in the organ recipient led to a late diagnosis. A blood film is simple and cheap to perform and may be life saving. We therefore re-emphasise the need to screen all febrile patients who have recently had a transplant operation (or transfusion) for malaria.

Pretransplantation evaluation of donors, especially a detailed travel history, is important but often difficult. An asymptomatic carrier from an area endemic for malaria may, however, be a high risk donor. We recommend systematic screening of donors, even after transplantation. A positive finding on malaria testing before transplantation need not prevent the transplantation but should protect both patients and clinicians against the hazards of diagnostic delay.

We thank Pr Grandjbakhch, department of heart transplantation, Pitié-Salpêtrière Hospital, and S Dussolier, T Seror, M Sibony, $\mathrm{N}$ Uwechue, and J Zanoni.

1 Holzer B, Glück Z, Zambelli D, Fey M. Transmission of malaria by rena transplantation. Transplantation 1984:39:315-6.

2 Johnston I. Possible transmission of malaria by renal transplantation. BMJ 1981;282:780.

3 Dharmasena F, Gordon-Smith EC. Transmission of malaria by bone marrow transplantation. Transplantation 1986;42:228.

4 Cruz I, Mody V, Callender C, Hosten A. Malaria infection in transplan recipient. F Natl Med Assoc C, Hos8;70:105-7.

5 Lefavour GS, Pierce JC, Frame JD. Renal transplant-associated malaria. ЭAMA 1980;244:1820-1.

(Accepted 20 fuly 1991)

\section{What happens to patients with non-vascular leg pain?}

\author{
K Varty, J van Dorpe, J A St Johnston, \\ W Bruce Campbell
}

\section{Department of Surgery, Royal Devon and Exeter Hospital, Exeter EX2 5DW $\mathrm{K}$ Varty, FRCS, senior house officer \\ J van Dorpe, MD, senior house officer \\ J A St Johnston, SRN, vascular research nurse W Bruce Campbell, Ms, consultant surgeon}

Correspondence to: Mr Campbell.

BMf 1991;303:1516

Conditions causing non-vascular leg pain in 13 patients, diagnosed after referral to other specialists

\begin{tabular}{ll}
\hline Diagnosis & No \\
\hline Spinal stenosis & 3 \\
Sciatica & 3 \\
Lumbar spondylosis & 2 \\
Peripheral neuropathy & 2 \\
Multiple sclerosis & 2 \\
Osteoarthritis of hip & 1 \\
Morton's neuroma & 1
\end{tabular}

$\star$ One patient had both spinal stenosis and peripheral neuropathy.
Non-vascular leg pain is a term used to describe pains in the leg or foot that suggest arterial occlusive disease but where specialist assessment shows that this is not the cause. Spinal claudication that mimics intermittent claudication is well recognised, ${ }^{1}$ but other conditions such as arthritis, neuropathy, and lymphoedema can also present as "atypical claudication." The diagnosis may be complicated by evidence of arterial disease that is not severe enough to account for the symptoms. Little is known about what happens to patients with non-vascular leg pain. We present a prospective study of such patients, assessed at least one year after they had initially been referred.

\section{Patients, methods, and results}

During the three years 1986-9, 525 patients with pain in the leg or foot were referred to the vascular surgical clinic. Among these, 55 had non-vascular pain (40 in the leg and 15 in the foot). We excluded diabetic foot problems. Arterial disease was excluded clinically and by measuring the systolic pressure at the ankle with Doppler ultrasonography and by performing selective stress testing with a simple heel raising exercise.

Patients were invited for review 12-48 (median 27) months later, and 49 attended. Their pain was assessed with a five point score (from "much better" to "much worse") and by linear analogue. The details of referrals to other specialists, the diagnoses that they had made, and the treatments that they had given were recorded. Quality of life was assessed by measuring on linear analogue scales the degree to which pain had interfered with sleep, activities in the home, work, and recreation. Ankle pressures were measured with Doppler ultrasonography before and after exercise testing. ${ }^{3}$

Fifteen patients had been referred to other specialists, who had made diagnoses in 13 (table). The eight patients with lumbar spine conditions were all treated conservatively. The two patients with multiple sclerosis had received injections of adrenocorticotrophic hormone. The patient with osteoarthritis of the hip had had a hip replacement operation. The symptoms of 11 of these patients were stable or improved.

The condition remained undiagnosed in 36 patients.
Symptoms were improved in 11 , unchanged in 17 , and worse in eight. Pain scores were significantly lower and quality of life scores significantly higher in these 36 patients than in the 13 patients who had been investigated and in whom a diagnosis had been made (mean pain score $3.60 v 5.27$ (95\% confidence interval 0.08 to 3.3$)$; mean quality of life score $77.7 v 59.0(2.5$ to $35 \cdot 0)$ ).

Six patients had evidence of mild arterial disease at follow up (a new finding in two), but none of these patients had symptoms that were compatible with arterial insufficiency.

\section{Comment}

Our finding that one in 10 patients referred with suspected arterial disease had non-vascular leg pain is similar to the experience of Tait et al. ${ }^{2}$ The follow up assessment one to four years after referral showed that Doppler measurements of pressure combined with selective exercise testing was effective in excluding arterial disease.

Problems of the lumbar spine are commonly confused with intermittent claudication, and symptoms need to be carefully evaluated to distinguish between these two conditions. ${ }^{14}$ It is rare for multiple sclerosis to present with symptoms mimicking vascular disease, but this happened in two of our patients.

This study identified two groups of patients, and the symptomatic outcome in each was generally good. The smaller group of patients with more troublesome symptoms were referred to other specialists. After treatment, which was largely non-surgical, only four patients were worse than before. The remaining patients had no further investigations, and at follow up less than a quarter said that their symptoms had deteriorated. No patient was severely disabled by pain.

After important arterial disease has been excluded by Doppler studies most patients with non-vascular leg pain can simply be reassured. Only patients whose symptoms are troublesome or worsen are likely to benefit from further investigation.

1 Stanton PE, Rosenthal D, Clark M, Vo N, Lamis P. Differentiation of vascular
and neurogenic claudication. Am Surg 1987;53:71-6.
2 Tait WF, Charlesworth D, Lemon JG. Atypical claudication. Br $\mathcal{F}$ Surg
1985;72:315-6.
3 Carter SA. Response of ankle systolic pressure to leg exercise in mild or
questionable arterial disease. N Engl $\mathcal{F}$ Med 1972;287:749-57.
4 Goodreau JJ, Creasy JK, Flanigan DP, Burnham SJ, Kudrna JC, Shafer MF,
et al. Rational approach to the differentiation of vascular and neurogenic
claudication. Surgery 1978;84:749-57.
5 Smigiel M, Davis DH. Pseudoclaudication: a review of etiology, diagnosis and Smigiel M, Davis DH. Pseudoclaudication: a review of etiolog
treatment. Geriatric Clinics of North America 1985;1:373-80.

(Accepted 4 fuly 1991) 